\title{
Isoflavone Content of Kansas Soybeans
}

\author{
M. Swanson ${ }^{1}$, M. Stoll ${ }^{2}$, W. Schapaugh ${ }^{2}$, and L. Takemoto ${ }^{1}$ \\ ${ }^{1}$ Division of Biology and ${ }^{2}$ Department of Agronomy \\ Kansas State University \\ Manhattan, Kansas 66506 USA
}

Received:September 30, 2003

Accepted: February 25, 2004

\begin{abstract}
Isoflavones occurring in soybean seed have been thought to have a positive effect on the health of humans, especially in cancer prevention. This positive impact may be a result of their antioxidant properties, estrogenic activity, or a combination of mechanisms. The purpose of this study was to quantify and compare different levels of isoflavones in various Kansas soybean cultivars. Seeds of four different cultivars grown in 2001 at three different locations were analyzed. There were significant differences in total and individual amounts of isoflavones across both cultivars and locations. These results demonstrated a large variation in isoflavone content in different cultivars grown in different locations, suggesting that selection of cultivar and growing location are important parameters in optimizing the isoflavone content of soybeans grown in Kansas.
\end{abstract}

\section{INTRODUCTION}

There are naturally occurring compounds found in plants thought to have certain cancer prevention properties. One class of these compounds, called isoflavones, has been found in large quantities in soybean. These soybean isoflavone phytoestrogens are plant-derived nonsteroidal estrogen mimics. Their roles in the biology of the soybean are not exactly clear. Their prevalence may explain why some Asian countries have a low incidence of certain cancers since soybean, and the accompanying isoflavones, are a large part of their diet. These isoflavones seem to have many mechanisms of action for cancer prevention, including: induction of cell-cycle arrest and apoptosis, prevention of oxidation, regulation of the host immune system, and estrogenic activity [1].

Isoflavones come in three parent forms: daidzein, glycitein, and genistein. Each parent isoflavone can be found in three chemical forms: the glucoside form, the acetyl glucoside ester form, and the malonyl glucoside ester form. Standards for the acetyl and malonyl glucoside esters are reportedly unstable in solution [2]. Thus, we employed a saponification step to eliminate the need for these standards by converting the malonyl and acetyl glucoside esters to the stable isoflavone glucoside [3]. The parent isoflavones were not quantified in this study due to the small amounts occurring in the soybeans and the small peaks that they produced on the HPLC. Analyzing such small peaks would have made the chance for error quite high. In this manner, it was possible to accurately determine total isoflavone content, while still being able to quantify the amounts of the glucoside derivatives in each soybean sample.

Tsukamoto et al. [4], have already studied the environmental effects on isoflavone concentration in soybeans. They found that isoflavone content was significantly lower in seeds that developed under high temperature conditions during seed fill than in seeds that developed under lower temperatures. They found that the cotyledon contained $80-90 \%$ of the total isoflavones of the soybean, with the rest being located in the hypocotyl. Also, the cotyledons exhibited a large response to different temperatures by producing different 


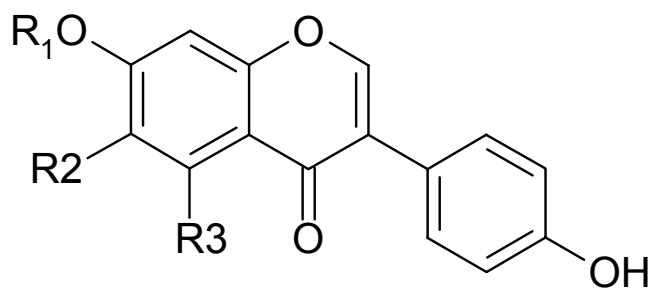

\begin{tabular}{|l|l|l|l|}
\hline Isoflavone & $\mathbf{R}_{\mathbf{1}}$ & $\mathbf{R}_{\mathbf{2}}$ & $\mathbf{R}_{\mathbf{3}}$ \\
\hline Daidzein & $-\mathrm{H}$ & $-\mathrm{H}$ & $-\mathrm{H}$ \\
\hline Glycitein & $-\mathrm{H}$ & $-\mathrm{OCH}_{3}$ & $-\mathrm{H}$ \\
\hline Genistein & $-\mathrm{H}$ & $-\mathrm{H}$ & $-\mathrm{OH}$ \\
\hline Daidzin & - Glucose & $-\mathrm{H}$ & $-\mathrm{H}$ \\
\hline Glycitin & - Glucose & $-\mathrm{OCH}_{3}$ & $-\mathrm{H}$ \\
\hline Genistin & - Glucose & $-\mathrm{H}$ & $-\mathrm{OH}$ \\
\hline
\end{tabular}

Figure 1. An illustration of the differences between the different isoflavone glucosides and the parent isoflavones.

levels of isoflavones, while the isoflavone content of the hypocotyl remained relatively constant across different temperatures [4].

Kansas ranks tenth in soybean production in the United States [5]. In addition, soybeans are the fourth largest cash crop in the state [5]. Therefore, soybean cultivars with both high protein yield and high isoflavone content would be expected to be very important in the economy of the state, especially in the development of niche markets for human consumption. While Hoeck et al. [6], have already studied the influence of genotype and environment on isoflavone content of soybeans, their study was limited to soybeans produced in lowa. The purpose of the present study was to determine isoflavone content in different cultivars and locations in Kansas soybeans within the same year. These cultivars have been chosen because of their high yields when grown in Northeastern Kansas. In addition, we wanted to analyze cultivars not previously characterized in other studies, to maximize the number of cultivars that could be candidates for future breeding projects to produce cultivars of high isoflavone content. The results of the present study demonstrate cultivar type and location of growth both have large effects upon the isoflavone content of soybeans grown in Northeast Kansas.

\section{EXPERIMENTAL}

The four cultivars used in this project were each grown in three different locations close to Manhattan, in Northeastern Kansas. Two of these locations were dryland, the Manhattan and Seneca locations, while the Ashland location was irrigated. All cultivars evaluated were non-GMO genotypes. One of the cultivars, KS4202, was produced for the general crushing market. The other three genotypes, KS4302sp, KS4402sp, and KS 4702sp were categorized as specialty food market cultivars with higher protein content than average soybeans. The seed from each cultivar and location was analyzed for isoflavone content. Isoflavones measured were daidzin, genistin, and glycitin. These standards were acquired from LC laboratories (Woburn, MA).

Whole seeds from each cultivar were weighed, and then dehydrated to a constant weight in a vacuum. All samples were ground to a powder in a standard laboratory mill containing ball bearings. Total isoflavones were extracted as described by Klump [3]. One gram of this ground soybean seed was placed into a $250 \mathrm{ml}$ Erlenmeyer 
flask and $40 \mathrm{ml}$ of $80 \%$ ( $\mathrm{vol} / \mathrm{vol}$ ) methanol in water was added to the ground seed. The flask was covered with parafilm and aluminum foil and placed in an orbital platform shaker and shaken at $65^{\circ} \mathrm{C}$ for two hours. The flask was then cooled to room temperature and $3 \mathrm{ml}$ of $2 \mathrm{M}$ sodium hydroxide was added. This solution was placed on a shaker and shaken again for 10 minutes at room temperature. One $\mathrm{ml}$ of glacial acetic acid was then added. The resulting solution was filtered through Whatman No. 42 filter paper into a $250 \mathrm{ml}$ beaker. Five $\mathrm{ml}$ of this filtrate was then mixed with $4 \mathrm{ml}$ of water and $1 \mathrm{ml}$ of the $80 \%$ (vol/vol) methanol in water.

Depending on the concentration of isoflavones, between $15-45 \mu \mathrm{L}$ of this mixture was analyzed using a $\mathrm{C}_{18}$ reversephase column, connected to a Beckman high performance liquid chromatography (HPLC) system. The column used in the HPLC analysis was made by Grace Vydac,

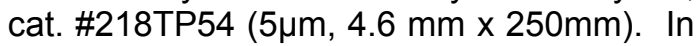
the HPLC analysis, solvent $A$ was $0.1 \%$ (vol/vol) glacial acetic acid in $\mathrm{H}_{2} \mathrm{O}$, and solvent $B$ was $0.1 \%(\mathrm{vol} / \mathrm{vol})$ glacial acetic acid in acetonitrile. The linear gradient was $10-45 \%$ of solvent B over 60 minutes and the flow rate was $1 \mathrm{ml} / \mathrm{min}$. Absorbance was monitored at 215 nanometers. Figure 2 shows that this gradient clearly separates the three different classes of isoflavones. Standards were also run on this gradient to help determine a standard curve to aid in quantitation. The experimental procedure was run four times per sample from each cultivar and location and the mean of these four replications were used in all statistical analyses.
The SAS, PROC GLM procedure was used for the analysis of variance of isoflavone concentrations [7]. Location and cultivar served as the main effects. Cultivar effects were considered fixed while location and the location by cultivar interaction were considered random effects. Treatment means were separated using Fisher's LSD at the 0.05 level of probability.

\section{RESULTS}

Table 1 shows the isoflavone content of different cultivars, grown in all three locations. This table shows only the three isoflavone glucoside amounts since the other two forms were saponified to those forms and are included in the totals. The parental forms were not analyzed since it is in our experience and the experiences of others that the parental forms are not present in high enough amounts to have much of an effect on the overall total of isoflavones [6]. We initially tried to separate the parental forms, but their amounts were too low for detection. For each cultivar, this analysis took the average of each isoflavone from the three locations (Ashland, Manhattan, and Seneca). A two and three fold difference in isoflavone concentrations was noted among the cultivars for daidzin and glycitin, respectively. The cultivars did not differ significantly in genistin concentration. Three of the cultivars (KS4202, KS4302sp and KS4702sp) contained similar levels of total than cultivar KS4402sp. Although cultivars 4202, 4302sp, and 4702sp contained similar amounts of total isoflavones, they did differ in daidzin and glycitin concentrations.

\begin{tabular}{lccccc}
\hline Isoflavone & $\begin{array}{c}\text { KS4202 } \\
(\mu \mathrm{g} / \mathrm{g})\end{array}$ & $\begin{array}{c}\text { KS4302sp } \\
(\mu \mathrm{g} / \mathrm{g})\end{array}$ & $\begin{array}{c}\text { KS4402sp } \\
(\mu \mathrm{g} / \mathrm{g})\end{array}$ & $\begin{array}{c}\text { KS4702sp } \\
(\mu \mathrm{g} / \mathrm{g})\end{array}$ & LSD $_{(0.05)}$ \\
\hline Daidzin & 677 & 337 & 363 & 619 & 157 \\
Glycitin & 227 & 182 & 78 & 164 & 22 \\
Genistin & 1111 & 1401 & 863 & 1152 & $\mathrm{~ns}$ \\
Total & 2015 & 1919 & 1303 & 1934 & 534 \\
\hline
\end{tabular}

Table 1. Isoflavone Content of Soybean Cultivars. 


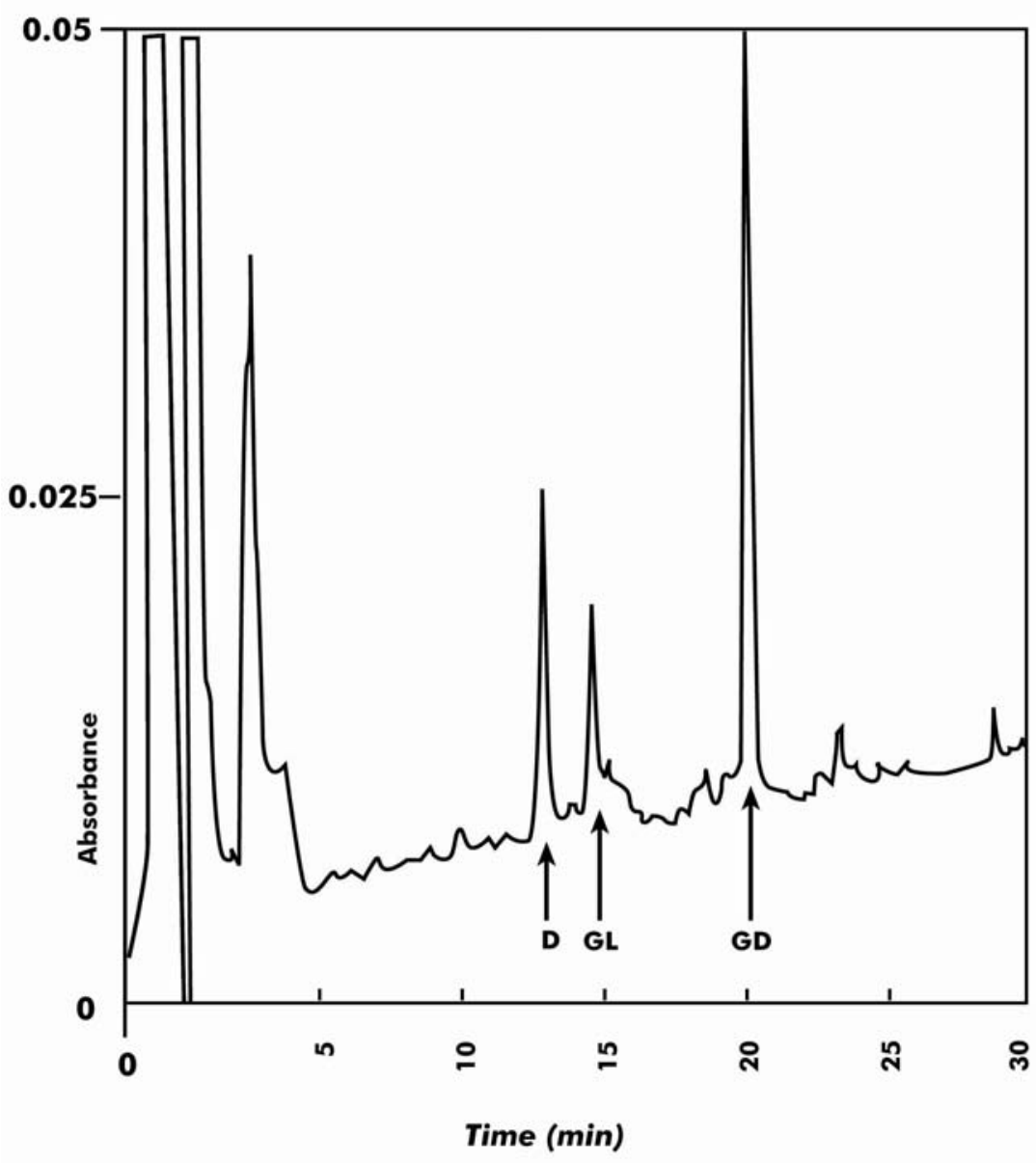

Figure 2. This illustrates a typical elution profile for a sample. The glucoside forms of the three isoflavones daidzein (D), genistein (GD), and glycitein (GL) are clearly separated by the gradient.

Table 2 shows the effect of location upon levels of isoflavones from all four cultivars. For each location, this analysis took the average of each isoflavone from the four cultivars (KS4202, KS4302sp, KS4402sp, and KS4702sp). Individual and total isoflavone concentrations differed among the locations by as much as $36 \%$. Samples from the Manhattan and Seneca locations had the highest total amounts of isoflavones, with the seeds collected from Manhattan having significantly greater amounts of total isoflavones than samples from both the Ashland and Seneca sites. It is interesting to note that the Ashland location was the only irrigated location. It is not clear from this data if irrigation or yield potential strongly influences the amount of each isoflavone type present in the seed, but certainly, location and/or environment do influence isoflavone makeup.

\section{DISCUSSION}

Isoflavones have many health
benefits, including their antioxidant properties, induction of cell-cycle arrest and apoptosis, regulation of host immune system, and induction of detoxification enzymes [1]. Many of these mechanisms are thought to play a role in the anti-cancer properties associated with soybeans. Two especially important mechanisms are the antioxidant properties and induction of cellcycle arrest and apoptosis. Antioxidants have been thought to prevent cancer by quenching free radicals, which are thought to induce covalent damage to both cell 


\begin{tabular}{lcccc}
\hline Isoflavone & $\begin{array}{c}2001 \text { Manhattan } \\
(\mu \mathrm{g} / \mathrm{g})\end{array}$ & $\begin{array}{c}2001 \text { Ashland } \\
(\mu \mathrm{g} / \mathrm{g})\end{array}$ & $\begin{array}{c}2001 \text { Seneca } \\
(\mu \mathrm{g} / \mathrm{g})\end{array}$ & LSD $_{(0.05)}$ \\
\hline Daidzin & 637 & 403 & 456 & 136 \\
Glycitin & 182 & 150 & 155 & 19 \\
Genistin & 1427 & 925 & 1043 & 382 \\
Total & 2246 & 1478 & 1654 & 462 \\
\hline
\end{tabular}

Table 2. Isoflavone Content In Soybean Across Locations.

membranes and cellular DNA. The cumulative effects of free radical damage may eventually lead to cancer and other health problems. Antioxidants may help prevent cancer by quenching these free radicals and thus preventing the cell damage from occurring.

Cell-cycle arrest and apoptosis are also important in preventing cancer. In the progression of cancer, apoptosis is many times turned-off, resulting in immortalized cancer cells. When major mutations occur in normal cells, apoptosis occurs and the cell dies. In cancer cells, this programmed cell death doesn't occur, leaving the cancer cells to propagate and cause damage to the patient. Antioxidants seem to play an important role in cancer prevention by inducing apoptosis in cancer cells and thus preventing the cancer cells from causing more damage [1]. Since the antioxidant properties of cells can be augmented by dietary intake, a strategy for the prevention of cancer would include a diet high in compounds with antioxidant properties such as isoflavones. An excellent dietary source of isoflavones is the soybean, which has been consumed in large quantities in Asian countries for centuries. The ratios of certain isoflavones in soybeans may also be important. Some preliminary studies have found that daidzin may not have much effect on humans and thus would not be as important when screening soybeans for isoflavone content [2]. Ratio of different isoflavone types would then become another important parameter to study when analyzing isoflavones.

In recent years, the beneficial effects of soybean consumption have become more known to the American public. Together with continued efforts to breed cultivars with high protein content, it is becoming increasingly important to access both the protein yield and antioxidant properties of these new cultivars, especially in the region of Northeastern Kansas where soybeans are a major crop. The results of the present study demonstrate that there were large variations in the isoflavone content of soybeans grown in this region. This conclusion is in marked contrast to the relatively small variations seen in protein content of soybeans grown in the same region (W. Schapaugh, results not published).

There are many possibilities as to why these different cultivars of soybeans had significant differences in isoflavone content. Our study seems to indicate that water availability may have some role in determining the amounts of isoflavones produced. Also, we have observed that there are differences among cultivars for isoflavone content. Due to unknown genetic factors, isoflavone content could be an intrinsic parameter that varies more greatly than other chemical contents of the soybeans. Future characterization of the genotypes of each cultivar would greatly assist in ascertaining this possibility.

The large variation in isoflavone content from different locations could have been influenced by a number of factors. The locations differed in soil types, which may influence fertility and water holding capacity. The planting dates of the cultivars differed by almost 30 days from the earliest to the latest planted trial, thus influencing the timing of vegetative and reproductive 
development throughout the growing season. All entries at one location were irrigated and the other two locations were produced dryland. All of these factors can dramatically influence the yield potential and amount of heat and drought stress experienced by a crop. Increased synthesis of antioxidants such as isoflavones may be a response to stress conditions possibly induced by dry growing conditions. Perhaps conditions of low soil moisture induce high isoflavone production, since these stress conditions have been known to cause a decrease in the redox potential of plant cells. Further studies are necessary to ascertain this intriguing possibility.

In conclusion, this study analyzed the average isoflavone content of different cultivars at the same location and the average isoflavone content of the same cultivar at different locations. In different cultivars at the same location, and the same cultivar at different locations, significant differences were observed in both the total levels of isoflavones and in levels of individual isoflavones, demonstrating that isoflavone content of soybeans is a highly variable quantity subject to multiple parameters that must be carefully chosen to ensure maximum productivity.

\section{REFERENCES}

1. D.F. Birt, S. Hendrich, and W. Wang. Pharm. \& Thera., 90, 157-177 (2001).

2. LC Laboratories 2003 Catalog, Soybean Isoflavone Standards and General Information, www.LCLabs.com, pp 3-7.

3. S.P. Klump, M.C. Allred, J.C. MacDonald, and J.M. Ballam. J. AOAC Int., 84, 1865-1883 (2001).

4. C. Tsukamoto, S. Shimada, K. Igita, S. Kudou, M. Kokubun, K. Okubo, and K. Kitamura. J. Agric. Food Chem., 42, 1184-1192 (1995).

5. USDA Crop Production 2002 Summary, usda.mannlib.cornell.edu/reports/nass r/field/pcp-bban/cropan03.pdf, pp 3435 (2003).

6. J.A. Hoeck, W.R. Fehr, P.A. Murphy, and G.A. Welke. Crop Sci., 40, 48-51 (2000).
7. SAS Institute. 1990. SAS Procedures Guide Release, 6.04 ed. SAS Institute, Cary, NC. 